\title{
Tensile Strength of Single Collagen Fibrils Isolated from Tendons
}

\author{
Noritaka Yamamoto \\ Biomechanics Laboratory, Department of Mechanical Engineering, Ritsumeikan University, Shiga, Japan
}

\section{Email address:}

noritaka@se.ritsumei.ac.jp

\section{To cite this article:}

Noritaka Yamamoto. Tensile Strength of Single Collagen Fibrils Isolated from Tendons. European Journal of Biophysics. Vol. 5, No. 1, 2017, pp. 1-6. doi: 10.11648/j.ejb.20170501.11

Received: November 28, 2016; Accepted: January 7, 2017; Published: February 16, 2017

\begin{abstract}
Tensile failure properties of single collagen fibrils were determined using our original tensile test method. Fibrils were directly isolated from the fascicles of mouse tail tendons. Both the ends of each fibril were wound onto the tips of two microneedles several times using micromanipulators. The fibril and tips were immersed in physiological saline solution. Then, the fibril was stretched to failure by moving the one microneedle. During tensile testing, the fibril was firmly attached to the tips of the microneedles, and broken between the tips with no slippage observed. The diameter of tested 10 fibrils was $410 \pm 60$ $\mathrm{nm}(\mathrm{Mean} \pm$ S.D.). The stress-strain curves of these fibrils were almost linear. Their tensile strength and failure strain were $100 \pm 32 \mathrm{MPa}$ and $34 \pm 11 \%$, respectively. These values were approximately 480 and $190 \%$ of those of the fascicles with the diameter of $81 \pm 12 \mu \mathrm{m}$, respectively.
\end{abstract}

Keywords: Collagen, Fibril, Tendon, Mechanical Properties, Tensile Strength

\section{Introduction}

Tendons have such hierarchical structures as collagen fascicles (80-320 $\mu \mathrm{m}$ diameter $)$, fibers $(\sim 1 \mu \mathrm{m}$ diameter $)$, fibrils (50-500 $\mathrm{nm}$ diameter), subfibrils (10-20 nm diameter), microfibrils $(\sim 3.5 \mathrm{~nm}$ diameter $)$, and molecules $(\sim 1.5 \mathrm{~nm}$ diameter and $\sim 300 \mathrm{~nm}$ length) [1-3]. On the surface of the fibrils, the periodic banding of the interval of $\sim 67 \mathrm{~nm}$ was observed because of the quarter staggered arrangement of collagen molecules. The basic knowledge of their structures and mechanical properties is essential to the biomechanics of tendons.

Many studies have been performed on the mechanical properties of tendons, their fascicles, and their fibers [4-6]. At the fibrillar level, direct mechanical measurements have only recently become possible by atomic force microscopy (AFM) and microelectromechanical systems (MEMS). The mechanical properties of single collagen fibrils have been measured using AFM-based tensile [7-10], nanoindentation [11-19], and bending [20-22] tests, and MEMS-based tensile [23-25] tests.

The primary role of tendons is the transmission of contractile forces from muscle to bone. The large tensile forces are directly applied to the tendons, and often induce tendon ruptures. Therefore, the understanding of the tensile failure mechanism of tendons is essential to the prevention and treatment of tendon injury. On the study for fibrils, it is important to determine the tensile strength and failure strain defined as the stress and strain at failure by tensile testing, respectively. However, there were only a few investigations for the tensile failure properties of fibrils. Yang et al. [10] performed the AFM-based tensile test of the fibrils reconstituted from purified tendon collagen. Shen et al. [25] performed the MEMS-based tensile test of the fibrils isolated from the dermis of sea cucumber. To our knowledge, there have been no studies on the tensile failure properties of collagen fibrils directly isolated from tendons, except for the work done by Svensson et al. [8] using AFM-based tensile test.

The purpose of the present study is to determine the tensile failure properties of the single collagen fibrils directly isolated from mouse tail tendons. Using our original tensile test method, the collagen fibrils were stretched to failure, and their tensile strength and failure strain were determined.

\section{Materials and Methods}

\subsection{Isolation of Fibrils}

Tails of male DD-y mice age 4 weeks weighing 22.3-23.0 
$\mathrm{g}$ were wrapped in gauze moistened with physiological saline solution, covered with thin plastic film, and stored at $-40^{\circ} \mathrm{C}$. Tissue collection was approved by the animal care committee of Ritsumeikan University. Before tensile testing, the tails were thawed at room temperature. Fascicles with the diameter of about $100 \mu \mathrm{m}$ were resected from the tail tendons with a surgical knife and split along the longitudinal axis with two pairs of forceps. These fascicles were immersed in distilled water in a test tube and stirred for 1 hour with a test tube mixer to be loosened into a cottony state. A small amount of the cottony fibrils was picked up and spread on a glass slide siliconized with Sigmacote (Sigma-Aldrich, USA).

\subsection{AFM Imaging}

AFM imaging of isolated collagen fibrils was performed using an AFM (Nanocute, Hitachi High-Tech Science, Japan) in tapping mode. It was operated in air and at room temperature. On the surface of the fibrils, the typical periodic banding of the interval of about $70 \mathrm{~nm}$ was observed (Figure 1). It was confirmed that the isolation procedure did not induce any obvious damages on the surface of the fibrils.

\subsection{Tensile Test}

Two micromanipulators (MM-89, MMO-202N, Narishige, Japan) were mounted on an inverted dark-field microscope (IX71, Olympus, Japan) (Figure 2). For gripping a single fibril on the glass slide, two microneedles were fabricated of a glass rod (1 mm diameter; G-1000, Narishige) using a micropipette puller (PC-10, Narishige). Their tips were tapered down to the diameter of about $2 \mu \mathrm{m}$. These microneedles were connected to the micromanipulators. The microscopic images were recorded on a DVD recorder and analyzed using an image analyzer (XL-20, Olympus).

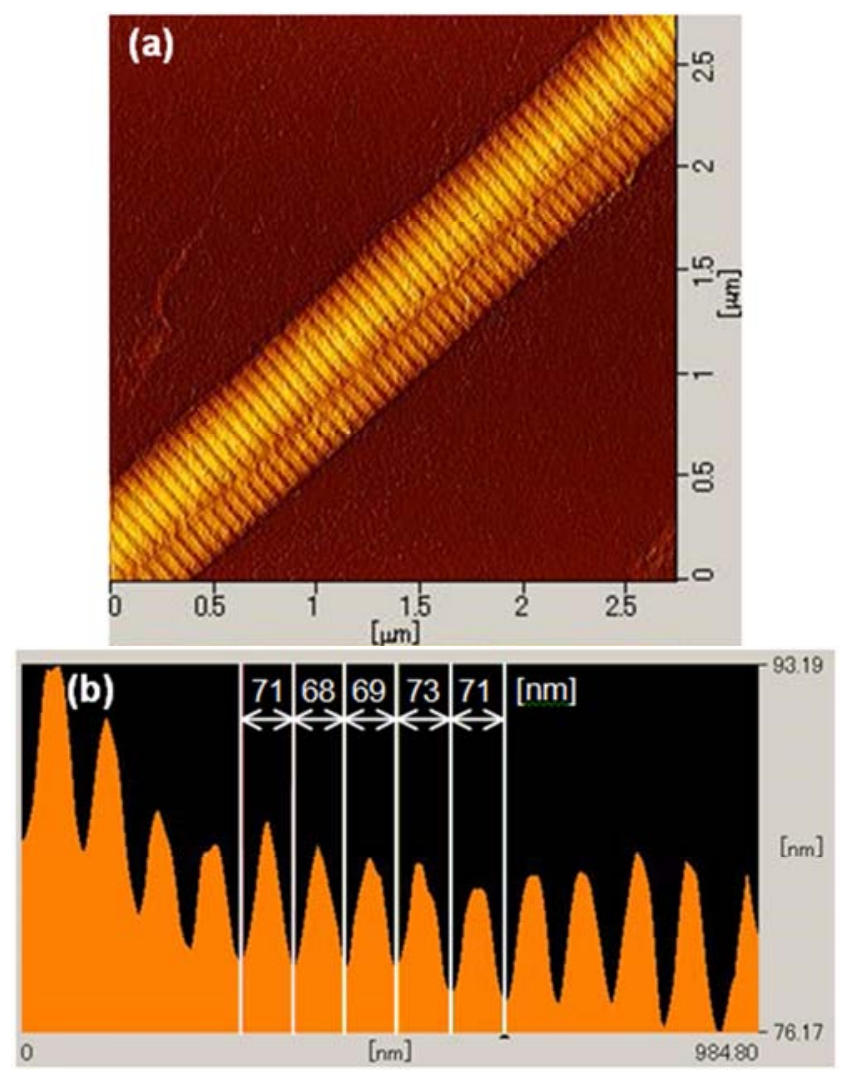

Figure 1. AFM images of an isolated collagen fibril. (a) The typical pattern of periodic banding was observed on the surface of the fibril. (b) The image of the cross section along the length of the fibril. The interval of the periodic banding was about $70 \mathrm{~nm}$.

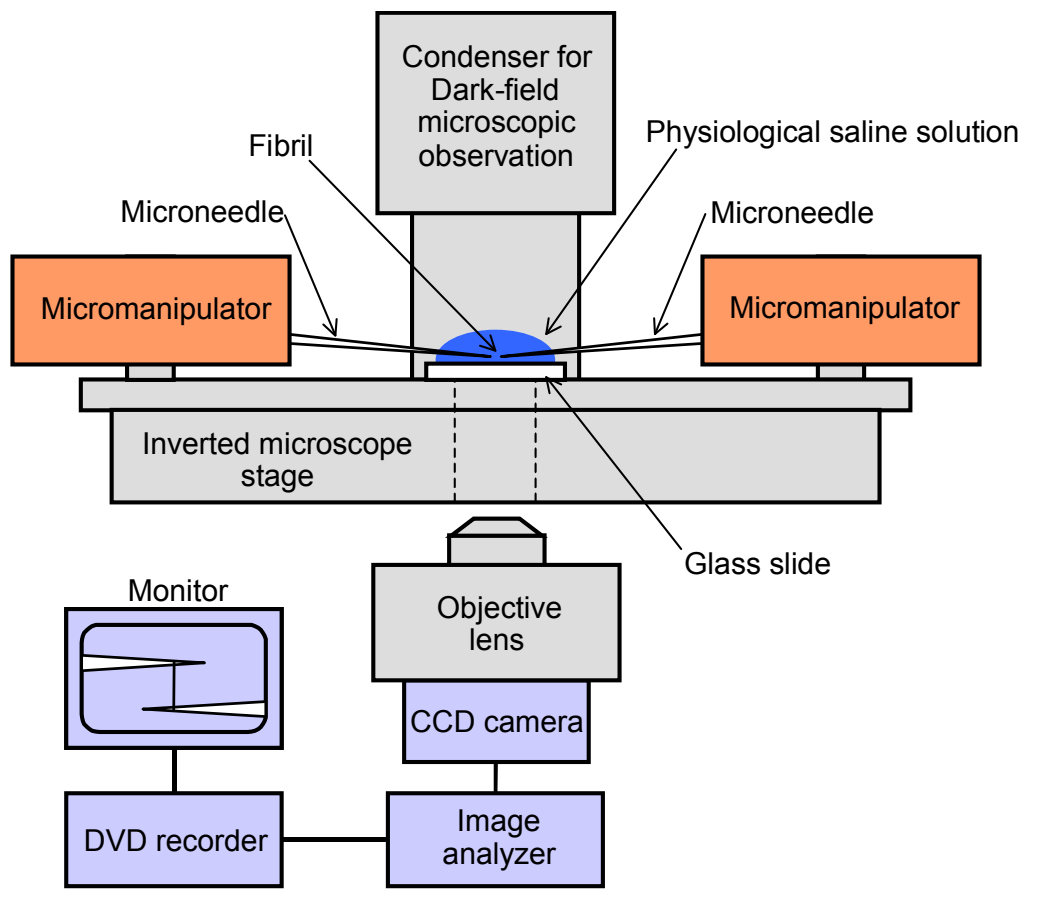

Figure 2. Schematic diagram of tensile tester. Two micromanipulators were mounted on an inverted dark-field microscope. For gripping a single fibril on the glass slide, two microneedles were connected to the micromanipulators. The microscopic images were recorded on a DVD recorder and analyzed using an image analyzer. 
One end of a single fibril was picked up from the glass slide with the tip of one microneedle (Figure 3(a)). The tip of the other microneedle was attached to the other end of the fibril (Figure 3(b)). The end of the fibril was wound to overlap each other onto the tip of the microneedle several times. And also, the other end of the fibril was wound onto the tip of the other microneedle (Figure 3(c)). The fibril and
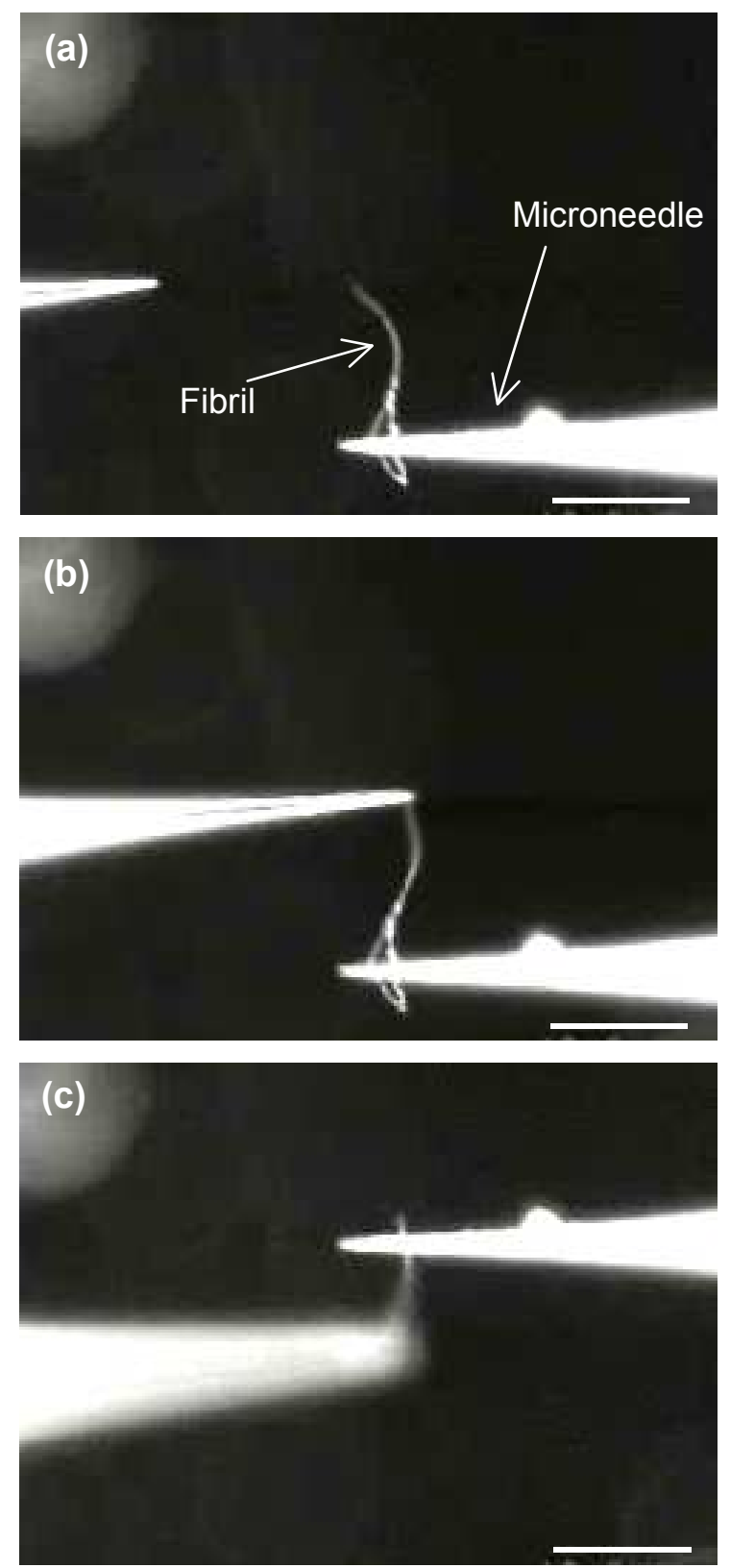

tips were immersed in physiological saline solution for 15 minutes (Figure 3(d)). Then, the fibril was stretched to failure by moving the one microneedle. The deflection of the microneedle and the length of the fibril were determined (Figure 3(e)). During tensile testing, the fibril was firmly attached to the tips of the microneedles, and broken between the tips with no slippage observed (Figure 3(f)).
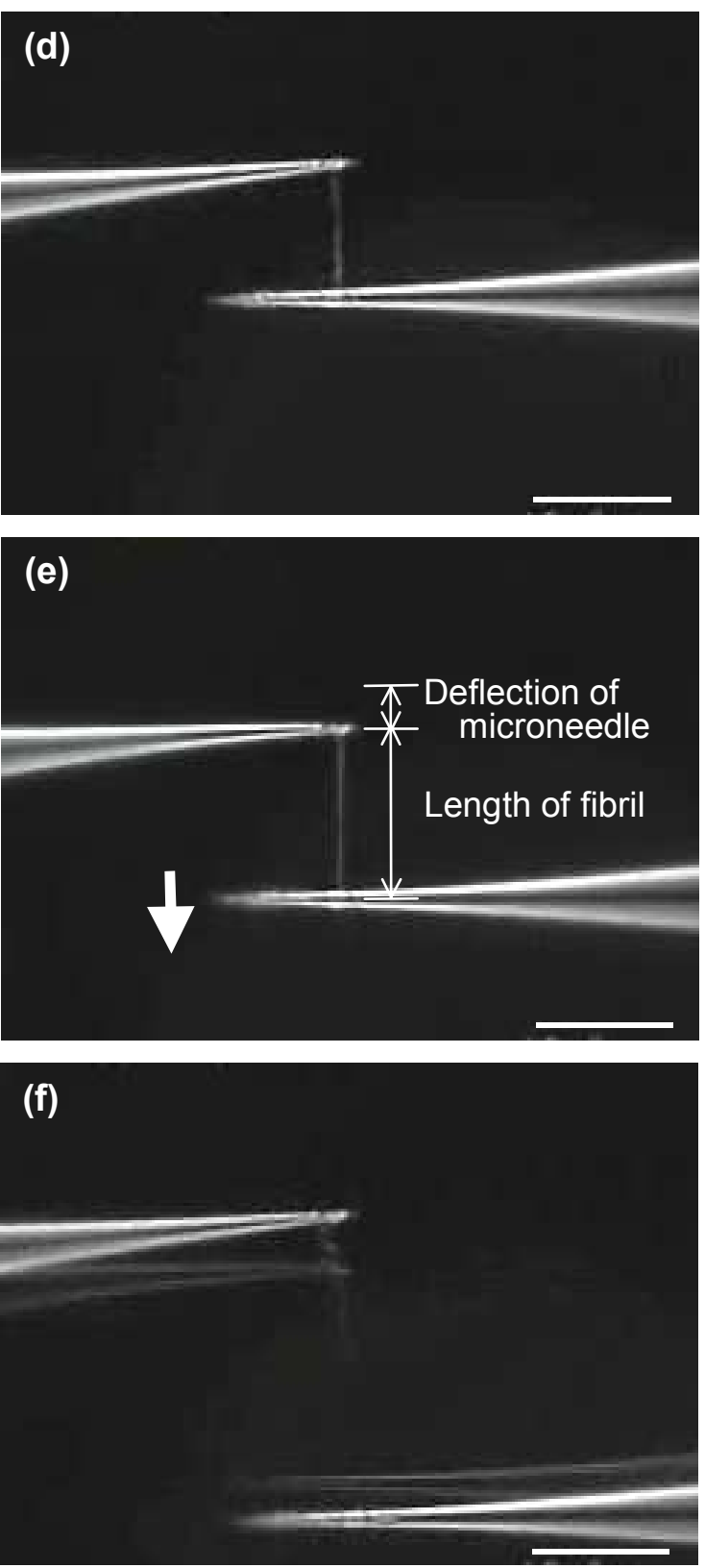

Figure 3. Schematic diagram of the procedure of tensile test. (a) One end of a single fibril was picked up from the glass slide with the tip of one microneedle. (b) The tip of the other microneedle was attached to the other end of the fibril. (c) The end of the fibril was wound to overlap each other onto the tip of the microneedle several times. And also, the other end of the fibril was wound onto the tip of the other microneedle. (d) The fibril and tips were immersed in physiological saline solution for 15 minutes. (e) The fibril was stretched to failure by moving the one microneedle. The deflection of the microneedle and the length of the fibril were determined. (f) The fibril was broken between the tips with no slippage observed. Scale bars $=50 \mu \mathrm{m}$.

\subsection{Measurement of the Cross-Sectional Area of Fibrils}

After the tensile test, the microneedles with the failed fibril were observed with a scanning electron microscope (SEM) and the diameter of the fibril was measured (Figure 4). The cross-sectional area was determined from the diameter, assuming that the cross section was circular. The average of cross-sectional areas at the ten positions was calculated as the representative of the cross-sectional area of the fibril. 


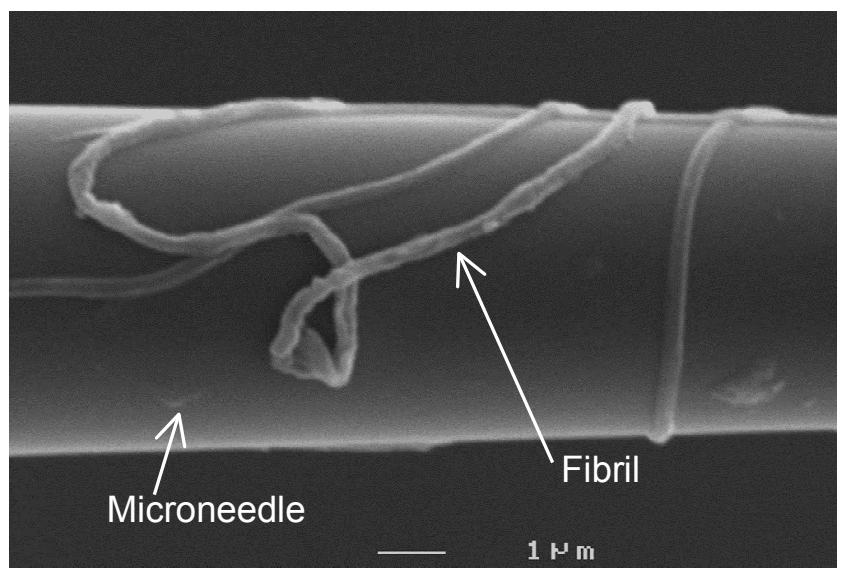

Figure 4. SEM image of a collagen fibril after tensile testing. The diameter of the fibril was measured. The cross-sectional area was determined from the diameter, assuming that the cross section was circular.

\subsection{Calibration of the Spring Constant of Microneedles}

The load applied to the fibril was determined from the deflection of the microneedle. Before the tensile test, the tip of the microneedle was pressed on the weighing pan of an ultra- microbalance (UMX2, Mettler-Toledo, Switzerland) using a micromanipulator. The deflection of the microneedle was measured using an image analyzer and the applied load was determined from the output of the ultra-microbalance. The load-deflection relation was linear, and the slope of the line (the spring constant of the microneedle) was $0.80 \pm 0.07 \mathrm{~N} / \mathrm{m}$.

\subsection{Calculation of Tensile Properties}

Stress was calculated by dividing the applied load by the cross-sectional area of the fibril. Strain was determined from dividing the deformation by the initial length of the fibril. From these data, the stress-strain relation was obtained. Tensile strength and failure strain were defined as the stress and strain at the failure point of the fibril, respectively.

\section{Results}

The diameter of tested 10 fibrils was $410 \pm 60 \mathrm{~nm}($ Mean $\pm \mathrm{S}$. D.). The averaged stress-strain relation of these fibrils was almost linear (Figure 5). Their tensile strength and failure strain were $100 \pm 32 \mathrm{MPa}$ and $34 \pm 11 \%$, respectively.

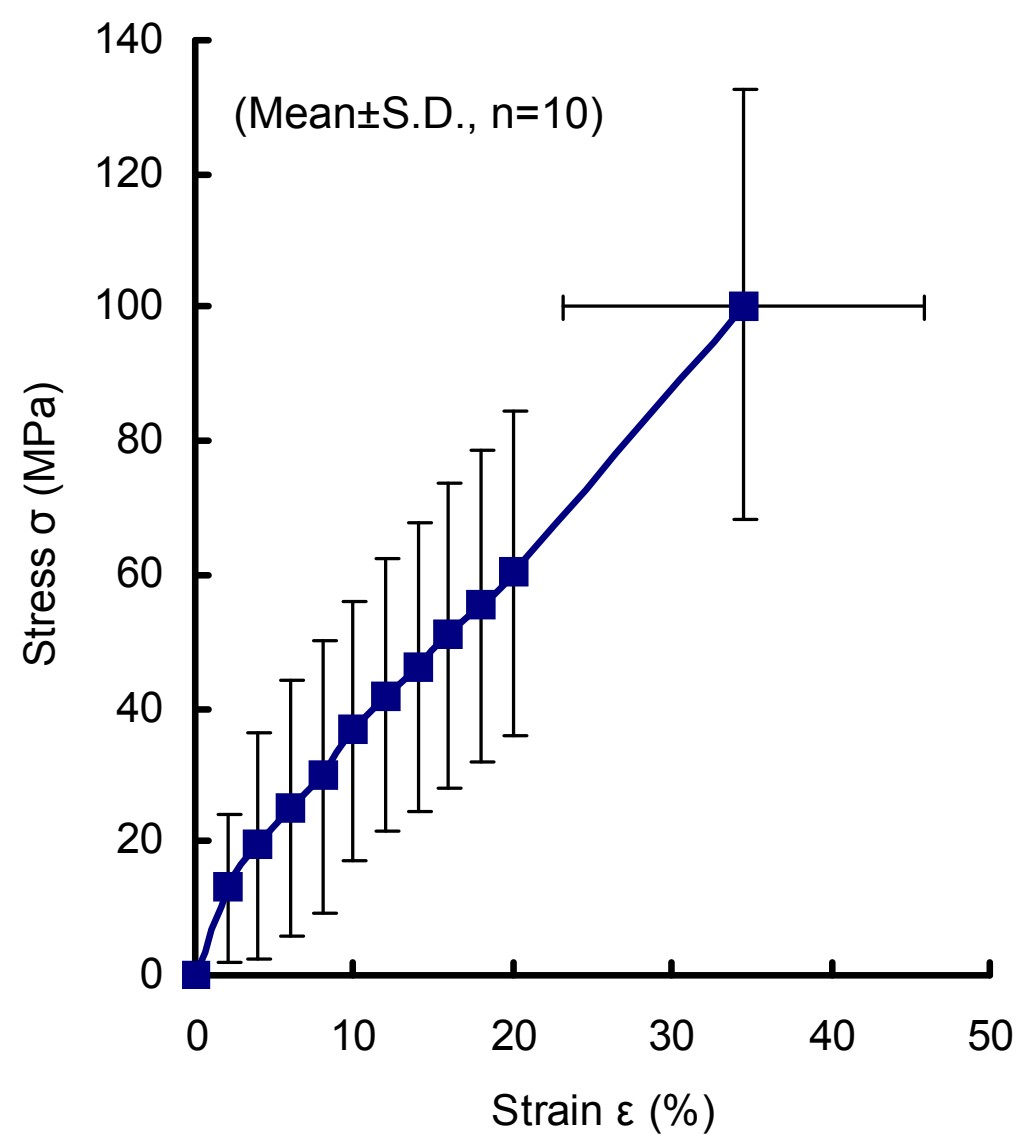

Figure 5. Averaged stress-strain relation of tested 10 collagen fibrils. This relation was almost linear. Their tensile strength and failure strain were $100 \pm 32$ MPa and $34 \pm 11 \%$, respectively.

\section{Discussion}

In the present study, the single collagen fibrils directly isolated from mouse tail tendons were stretched to failure in physiological saline solution using our original tensile test method. Their tensile strength and failure strain were determined. In this tensile test method, both the ends of each fibril were wound onto the tips of microneedles. This technique worked very well to grip the fibril. The adhesion 
between the tips and the fibril was strong enough to carry out tensile test. There was no slippage between the tips and the fibril, and the fibril was broken between the tips.

Svensson et al. [8] performed the AFM-based tensile test of the fibrils $(160 \pm 70 \mathrm{~nm}$ diameter) isolated from human patellar tendons, and reported that their tensile strength and failure strain were $540 \pm 140 \mathrm{MPa}$ and $20 \pm 1 \%$, respectively. In the same work by Svensson et al. [8], they reported that the tensile strength and failure strain of the fibrils $(210 \pm 150 \mathrm{~nm}$ diameter) isolated from rat tail tendons were $200 \pm 110 \mathrm{MPa}$ and $16 \pm 4 \%$, respectively. These tensile strength is larger than that of the present study. This difference may be attributable to the different diameter of fibrils. The diameter of fibrils in the present study was larger than that in the study by Svensson et al.. Fibrils are composed of subfibrils, and the subfibrils are arranged parallelly in the longitudinal direction [1-3]. Within larger-diameter fibrils, the orientation of subfibrils is disturbed, and the number of the longitudinally aligned subfibrils decreases. Therefore, the subfibrils are not evenly loaded, but the stress on some subfibrils may exceed their tensile strength before the overall mean stress does not. Such sequential breakage of the subfibrils would lead to the decrease of the tensile strength with increasing the diameter.

Yang et al. [10] performed the AFM-based tensile test of the fibrils reconstituted from purified bovine Achilles tendon collagen, and reported that their tensile strength and failure strain were $60 \pm 10 \mathrm{MPa}$ and $13 \pm 2 \%$, respectively. These results are smaller than those of the present study. These lower values may be due to the lack of cross-links in the reconstituted fibrils as compared with the fibrils directly isolated from tendons. In the same work by Yang et al. [10], cross-linking agents were found to increase the tensile strength and failure strain of the reconstituted fibrils.

Shen et al. [25] performed the MEMS-based tensile test of the fibrils isolated from the dermis of sea cucumber, and reported their tensile strength and failure strain were $230 \pm 160 \mathrm{MPa}$ and $80 \pm 44 \%$, respectively. These results are larger than those of the present study. It is difficult to compare the tensile properties of these fibrils between mammals and echinoderms because of their different structure [26].

In the present experiment, the diameter of dried fibrils was measured with SEM imaging after tensile testing. The crosssectional area of the dried fibrils was calculated from the diameter. And also, in the previous studies, the crosssectional area of dried fibrils was determined using AFM or SEM imaging $[8,10,25]$. On the other hand, the tensile tests of fibrils were performed in physiological saline solution. Stress was calculated by dividing the applied load by the cross-sectional area of the dried fibrils. Yang et al. [10] reported that the swelling of $\sim 50 \%$ in fibril diameter was found upon hydration in physiological saline solution using AFM imaging. It is possible that the decreased diameter of fibrils by drying may lead to the overestimation of stress.

We performed the tensile test of the fascicles $(81 \pm 12 \mu \mathrm{m}$ diameter) isolated from mouse tail tendons, and reported that their tensile strength and failure strain were $21 \pm 3 \mathrm{MPa}$ and
$18 \pm 4 \%$, respectively [27]. The tensile strength and failure strain of fibrils in the present study were approximately 480 and $190 \%$ of those of the fascicles, respectively. Fascicles consist of fibrils embedded in proteoglycans [1-3]. Therefore, fascicles may fail by the slippage and breakage between fibrils and proteoglycans rather than by the breakage of fibrils. These results indicate that the mechanical interactions between fibrils and proteoglycans contribute to the tensile properties of fascicles.

\section{Conclusion}

The single collagen fibrils directly isolated from the fascicles of mouse tail tendons were stretched to failure in physiological saline solution using our original tensile test method. The tensile strength and failure strain of these fibrils were much larger than those of fascicles.

\section{Acknowledgements}

This work was supported by JSPS KAKENHI Grant Number JP20560092. The author thanks Mr. Kazunari Sugiura for his experimental assistance.

\section{References}

[1] J. Kastelic, A. Galeski, and E. Baer, "The multicomposite structure of tendon”, Connect. Tissue Res., vol. 6, pp. 11-23, 1978.

[2] V. Ottani, D. Martini, M. Franchi, A. Ruggeri, and M. Raspanti, "Hierarchical structures in fibrillar collagens", Micron, vol. 33, pp. 587-596, 2002.

[3] F. H. Silver, J. W. Freeman, and G. P. Seehra, "Collagen selfassembly and the development of tendon mechanical properties", J. Biomech., vol. 36, pp. 1529-1553, 2003.

[4] N. Yamamoto, K. Hayashi, H. Kuriyama, K. Ohno, K. Yasuda, and K. Kaneda, "Mechanical properties of the rabbit patellar tendon", J. Biomech. Eng., vol. 114, pp. 332-337, 1992.

[5] E. Yamamoto, K. Hayashi, and N. Yamamoto, "Mechanical properties of collagen fascicles from the rabbit patellar tendon", J. Biomech. Eng., vol. 121, pp. 124-131, 1999.

[6] H. Miyazaki and K. Hayashi, "Tensile tests of collagen fibers obtained from the rabbit patellar tendon", Biomed. Microdevices, vol. 2, pp. 151-157, 1999.

[7] R. B. Svensson, T. Hassenkam, C. A. Grant, and S. P. Magnusson, "Tensile properties of human collagen fibrils and fascicles are insensitive to environmental salts", Biophys. J., vol. 99, pp. 4020-4027, 2010.

[8] R. B. Svensson, H. Mulder, V. Kovanen, and S. P. Magnusson, "Fracture mechanics of collagen fibrils: influence of natural cross-links", Biophys. J., vol. 104, pp. 2476-2484, 2013.

[9] J. A. van der Rijt, K. O. van der Werf, M. L. Bennink, P. J. Dijkstra, and J. Feijen, "Micromechanical testing of individual collagen fibrils", Macromol. Biosci., vol. 6, pp. 697-702, 2006. 
[10] L. Yang, K. O. van der Werf, P. J. Dijkstram, J. Feijen, and M. L. Bennink, "Micromechanical analysis of native and crosslinked collagen type I fibrils supports the existence of microfibrils", J. Mech. Behav. Biomed. Mater., vol. 6, pp. 148-158, 2012.

[11] C. A. Grant, D. J. Brockwell, S. E. Radford, and N. H. Thomson, "Effects of hydration on the mechanical response of individual collagen fibrils", Appl. Phys. Lett., vol. 92, pp. 233902-1-3, 2008.

[12] C. A. Grant, D. J. Brockwell, S. E. Radford, and N. H. Thomson, "Tuning the elastic modulus of hydrated collagen fibrils", Biophys. J., vol. 97, pp. 2985-2992, 2009.

[13] A. J. Heim, W. G. Matthews, and T. J. Koob, "Determination of the elastic modulus of native collagen fibrils via radial indentation", Appl. Phys. Lett., vol. 89, pp. 181902-1-3, 2006.

[14] S. Strasser, A. Zink, M. Janko, W. M. Heckl, and S. Thalhammer, "Structural investigations on native collagen type I fibrils using AFM", Biochem. Biophys. Res. Commun., vol. 354, pp. 27-32, 2007.

[15] M. P. Wenger, L. Bozec, M. A. Horton, and P. Mesquida, "Mechanical properties of collagen fibrils", Biophys. J., vol. 93, pp. 1255-1263, 2007.

[16] O. G. Andriotis, W. Manuyakorn, J. Zekonyte, O. L. Katsamenis, S. Fabri, P. H. Howarth, D. E. Davies, P. J. Thurner, "Nanomechanical assessment of human and murine collagen fibrils via atomic force microscopy cantilever-based nanoindentation”, J. Mech. Behav. Biomed. Mater., vol. 39, pp. 9-26, 2014.

[17] S. J. Baldwin, A. S. Quigley, C. Clegg, L. Kreplak, "Nanomechanical mapping of hydrated rat tail tendon collagen I fibrils", Biophys. J., vol. 107, pp. 1794-1801, 2014.

[18] S. J. Baldwin, L. Kreplak, J. M. Lee, "Characterization via atomic force microscopy of discrete plasticity in collagen fibrils from mechanically overloaded tendons: nano-scale structural changes mimic rope failure", J. Mech. Behav. Biomed. Mater., vol. 60, pp. 356-366, 2016.

[19] E. C. Spitzner, S. Roper, M. Zerson, A. Bernstein, R. Magerle, "Nanoscale swelling heterogeneities in type I collagen fibrils", ACS NANO, vol. 9, pp. 5683-5694, 2015.

[20] A. J. Heim, T. J. Koob, and W. G. Matthews, "Low strain nanomechanics of collagen fibrils", Biomacromolecules, vol. 8, pp. 3298-3301, 2007.

[21] L. Yang, K. O. van der Werf, B. F. Koopman, V. Subramaniam, M. L. Bennink, P. J. Dijkstra, and J. Feijen, "Micromechanical bending of single collagen fibrils using atomic force microscopy", J. Biomed. Mater. Res. A., vol. 82A, pp. 160-168, 2007.

[22] L. Yang, K. O. van der Werf, C. F. Fitie, M. L. Bennink, P. J. Dijkstra, and J. Feijen, "Mechanical properties of native and cross-linked type I collagen fibrils", Biophys. J., vol. 94, pp. 2204-2211, 2008.

[23] S. J. Eppell, B. N. Smith, H. Kahn, and R. Ballarini, "Nano measurements with micro-devices: mechanical properties of hydrated collagen fibrils", J. R. Soc. Interface, vol. 3, pp. 117 121, 2006.

[24] Z. L. Shen, M. R. Dodge, H. Kahn, R. Ballarini, and S. J. Eppell, "Stress-strain experiments on individual collagen fibrils", Biophys. J., vol. 95, pp. 3956-3963, 2008.

[25] Z. L. Shen, M. R. Dodge, H. Kahn, R. Ballarini, and S. J. Eppell, "In vitro fracture testing of submicron diameter collagen fibril specimens", Biophys. J., vol. 99, pp. 19861995, 2010.

[26] J. A. Trotter, F. A. Thurmond, and T. J. Koob, "Molecular structure and functional morphology of echinoderm collagen fibrils", Cell Tissue Res., vol. 275, pp. 451-458, 1994.

[27] N. Yamamoto and K. Sugiura, "Effects of ground substance on the mechanical properties of fascicles obtained from mouse tail tendons", Proc. 2nd Japan-Switzerland Workshop on Biomechanics, p. 4, 2005. 